\title{
The Use of Emoji in Electronic User Experience Questionnaire: An Exploratory Case Study
}

\author{
Sarah Alismail \\ Claremont Graduate University \\ Sarah.alismail@,cgu.edu
}

\author{
Hengwei Zhang \\ Claremont Graduate University \\ Hengwei.zhang@,cgu.edu
}

\begin{abstract}
Emojis are a growing phenomenon that have gained popularity in the digital era. However, little research exists on their use; a notable absent topic is the adoption of emojis in user experience questionnaires (UEQs). This study draws on semistructured interview data from 31 participants to understand how emojis affect the experience of filling out UEQs. The data analysis reveals two key categories regarding participants' experiences: advantages and challenges. This research contributes to the existing literature by presenting a detailed exploratory case study of user reactions to emojis in the context of UEQs.
\end{abstract}

\section{Introduction}

Emoji use is growing among people as a way to assist the process of conveying emotions in textual communication that lacks nonlinguistic cues [1]. Emojis were created as visual mixed expressions of sentiments, attitudes, or moods for use in modern communication technologies [2]. Emojis are a new phenomenon, so little research exists on their application, particularly in the context of user experience questionnaires (UEQ). Emojis may be used as a language-independent alternative to items that usually require a certain level of fluency in a specific language when making subjective assessments and expressing opinions. In the field of healthcare, for example, pictorial pain scale (emojis- like pictures) are used as a tool assisting in pain management during clinical sittings. In fact, several studies indicated the effectiveness of pictorial pain scale to be used with patients aged between 4 to 17 in emergency rooms as well as those who experience chronic pain [3]. Therefore, there may be a potential for using emojis in the context of UEQs. Kaye et al. [4], pointed out that emojis have the potential to be survey instruments such as tools for assessing personal perceptions and emotional expression. UEQ is a well-constructed and validated instrument to assess individuals' perception of anticipating/ using a product [5,6]. Offering a lens for understanding emojis and their implications for electronic UEQs makes for an intriguing area of new research.

The present study examines the potential of emojibased electronic questionnaires to improve the survey participation experience. This study draws from social cognitive theory (SCT), through which emojis can be understood as an existing phenomenon that gains growth and popularity in an external setting or environment. The study investigates how emojis can be applied in survey questionnaires to enhance participants' experiences in filling out UEQs. The research aims to answer the following question: How do emojis influence individuals' experiences in filling out electronic mobile application user experience survey questionnaires? To address the research question, an exploratory case study has been conducted to examine why participants have positive or negative experiences with emoji-based UEQs.

This study first presents a literature review of the emoji phenomenon and UEQs. Then, the applied research methodology and chosen modes for data analysis are described. Next, research findings and interpretations are introduced, followed by a discussion of the results and their implications. Finally, conclusions and recommendations for future work are presented.

\section{Literature review}

\subsection{Emojis}

An emoji is a two-dimensional pictograph used in modern communication technologies to assist expressive messages [7,8]. An emoji is "a graphic symbol, ideogram, that represents not only facial expressions, but also concepts and ideas ... emotions, feelings, and activities" (p.1) [7]. Emojis were developed in Japan by Shigetaka Kurita at the end of 
the 20th century to assist with digital communication. The word "emoji" stands for "picture character," in which the "E" means picture, "MO" means writing, and "JI" means character [7,9].

The frequency and popularity of emoji use has increased in recent years [10]. Oxford Dictionaries officially announced this type of pictograph as "Word of the Year" in 2015. The "face with tears of joy" emoji $\Leftrightarrow$ was chosen by Oxford Dictionaries to best reflect "the ethos, mood, and preoccupations of 2015" (p.1) [11].

Emojis become communicative signs because people imbue them with meaning through symbolism to communicate with each other. These images convey a complexity of emotions that cannot translate easily into words [12]. Bavelas and Chovil [13] describe visible acts of meaning for nonverbal behavior, a concept similar to emojis. Bavelas and Chovil [13] propose four criteria for visible acts of meaning as follows:

\section{...(a) visible acts of meaning are sensitive to a sender-receiver relationship; (b) these acts are symbolic, that is, they are being used to stand for something else; (c) their meaning can be explicated or demonstrated contextually; and (d) these acts are always integrated with the accompanying words, whether their meaning is redundant or nonredundeant with words ( $\mathrm{p}$. 168).}

Paul Ekman was one of the first psychologists to study the grammar of facial expressions, authoring an "atlas of emotions" that is currently used largely by semioticians and scientists [14]. Ekman observed that the basic emotions of "disgust, fear, anger, contempt, sadness, surprise, happiness" actuate the same microexpression patterns in a statistically predictable variation around the globe [14]. Although several factors - such as geographic location, age, and racecan affect how an individual manages these displays, Ekman and Friesen [15] suggest that the affect display of these seven emotions is recognizable both in familiar and foreign cultures. Danesi [14] asserts that people worldwide react similarly to basic facial emojis because "they incorporate the main micro-expressions in stylized outline form" [14].

\subsection{Emoji as a kinesic digital nonverbal codes}

The idea of kinesics was proposed by Ray Birdwhistell. He claimed that human body movements have meaning, and that nonverbal behavior has grammar structures that can possibly be analyzed like the spoken language $[16,17]$. Nonverbal codes are "clusters of behaviors that are used to convey meaning" (p.126) [18]. The term "digital nonverbal codes" refers to textual messaging used in mobilemediated communication [19]. Durante [19] further states that kinesic codes or emojis are digital substitutions for "facial expression, body language, gestures, and eye behavior" (p.16). Kinesics is but one of the seven nonverbal code functions proposed by Burgoon, Buller, and Woodall in 1996, which include facial expression, body movement, and gaze [19].

According to Durante [19], there are seven main functions of the non-verbal codes proposed by $[15,20$, 21]. According to these functions, digital kinesics or emojis can do the following:

1. Repeat what is said or sent;

2. Substitute a whole or part of a text;

3. Complement and clarify a text;

4. Contradict a text;

5. Emphasize or elaborate a certain text;

6. Accent or moderate a text; and

7. Regulate a written text.

\subsection{User experience(UX) and the UEQ}

According to ISO 9241-110:2010 [5], the term "user experience" refers to "a person's perceptions and responses that result from the use and/or anticipated use of a product, system or services." Simply put, the user experience describes how a person feels about using a product in particular conditions, including the experiential, affective, meaningful, and valuable aspects of product use (WIKI). Laurel [22] suggests that user experience focuses on individual perceptions (e.g., subjective usability, user-perceived quality). Several studies have identified a variety of aspectssuch as values, emotions, expectations, and prior experiences - that influence the experience evoked by user-product interaction [23-25].

UX generally describes the internal and emotional state of a person during and after an interaction with a product. This state has been described as "inherently dynamic" [26, 27]. To assess UX, experts develop specific tools, methods, and metrics [6,28-31] to measure the various aspects of the user experience that were described above. Questionnaires and surveys are common methods because they are easy to apply and simple to analyze [29]. Questionnaires use predefined standards to produce results that can be compared easily [32]. Many questionnaires use scales to distinguish the different intensities of answers. Others use predefined measures, such as the semantic differential questionnaire designed by [33], which uses a bipolar scale (i.e., opposite adjectives) to record participant reactions. 
Questionnaire designers must consider their target participants and the type of product being evaluated when selecting adjectives for semantic differentials. Laugwitz et al. [6] constructed and validated a 26-item semantic differential questionnaire that enables users of software products to assess the products in a simple and immediate way. This questionnaire includes six categories: attractiveness, perspicuity, efficiency, dependability, stimulation, and novelty. The advantage of this UEQ is its easy application, reliability, and valid measures of user experience, most significantly in the case of software products.

\subsection{Social cognitive theory}

SCT deals with how internal cognitions and environmental determinants function collectively to shape and control an individual's behavior [34]. The theory is based on the notion that humans learn by observing others. However, social learning theorists caution that learning may not necessarily lead to a change in an individual's behavior [35-37].

...New ideas, values, behavior patterns, and social practices are now being rapidly diffused worldwide by symbolic modeling in ways that foster a globally distributed consciousness (Bandura, 1986, 2001a). Because the symbolic environment occupies a major part of people's everyday lives, much of the social construction of reality and shaping of public consciousness occurs through electronic acculturation (p.27) [35].

SCT accounts for the reciprocal influences of personal agency, behavioral patterns, and environmental events (i.e., external settings) [34]. Thus, interaction occurs among these three determinants (i.e., personal, behavioral, and environmental) to influence the individual's behavior. Change occurs when the individual acquires an observed behavior [34]. People extensively regulated their motivation and action by forethought [38].

\section{Research methodology}

The present study aims to explore and understand people's experiences with emojis in UEQs. To do this, a qualitative research approach has been adopted, as this method allows the interviewed individuals' thoughts and feelings regarding their experiences with emoji-based UEQs to be collected effectively. An exploratory case study was conducted to understand the potential of emoji-based electronic questionnaires to improve user experiences. The case study is based on semi-structured interviews conducted by the authors. ${ }^{1}$

\subsection{Sample and procedure}

Semi-structured interviews were used to collect data from the participants on their experiences interacting with emojis in electronic UEQs. The interviews consisted of pre-formulated questions. Additional questions were generated adaptively during the interviews, depending on participant responses. In addition to the interviews, field notes were used to record contextual (situational and environmental) information necessary for interpreting the voicerecorded conversations.

A convenience sample of thirty-one participants, 13 male and 18 females, were selected from the general public in two cities in Southern California (e.g. people on the street or in coffee-shops). Participant ages ranged from 21 to 55 years old. The interviews took approximately 25 to 30 minutes to complete. Interviews were voice-recorded with verbal permission from the participants. One participant preferred not to be voice-recorded. Later, the interviews were transcribed into electronic text for analysis purposes.

Data collection occurred in three stages. First, the interviews began with the introduction of a mobile application from the Center of Disease Control and Prevention (CDC). This was a smartphone application designed to educate people on health topics via informative media such as stories and videos. Participants were given time to interact with the mobile app and explore its features until they felt confident to take the questionnaire.

In the second phase, a first version of the UEQ was given to the participants for them to assess their experience with the CDC mobile app via a tablet (see Appendix B). Originally, this version of the UEQ included a seven-point scale with a total of 26 items to measure perspicuity, efficiency, dependability, novelty, stimulation, and attractiveness. However, prior to the actual study, a pilot study with five participants was conducted to pre-test and ensure the feasibility, time, and protocol of the interview questions and the selected UEQ. Based on the feedback provided, the UEQ scale was reduced to five points (the seven-point scale was described as too confusing during the selection process in filling out the questionnaire). Thus, the UEQ selected in the formal study had only five points for consistency, and to make the points more distinct. Four items were removed as the pilot participants felt that they were redundant. Moreover,

\footnotetext{
${ }^{1}$ This study has been classified as an exempt research by the IRB
} 
"inferior", one of the semantic items, was replaced with the word "unvaluable" because the word "inferior" was described as incomprehensible. After completing the questionnaires, participants were asked semi-structured questions about their experiences with the scale-based questionnaire.

The third phase required participants to fill out a second, emoji-based version of the UEQ via a tablet (see Appendix C). Unlike the text version of the UEQ, which employed two columns of opposite adjectives, the emojis were organized on a single scale representing both positive and negative reactions. To accommodate this, the questionnaire had to be modified into a single column. Emojis were selected based on the provided "emojiscore" [39]. The emojiscore offered five IOS facial emojis on a scale from positive through neutral to negative for

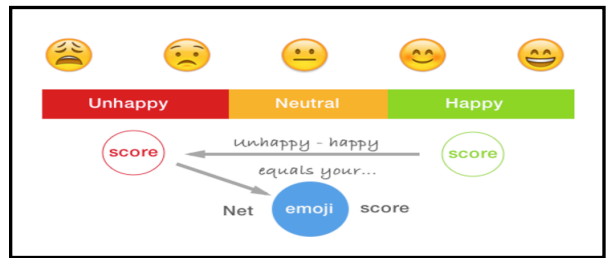

Figure 1. Five-scale emoji Source: Emojiscore

participants to choose from (see Figure 1). After the participants completed the survey, they were asked semi-structured questions about the experience.

\subsection{Data analysis}

Descriptive coding was used to conduct the data analysis. Miles and Huberman [40] define codes as

tags or labels for assigning units of meaning to the descriptive or inferential information compiled during a study. Codes . . . are attached to 'chunks' of varying size-words . . . connected or unconnected to a specific setting (p. 56).

A bottom-up approach was applied in the present study, meaning concepts and codes were generated from the analysis of the collected data. Textual data (e.g., sentences, paragraphs, and phrases) from the interview transcripts and field notes were analyzed and summarized with succinct codes. A continuous interplay between data collection and data analysis was maintained until saturation was reached.

\section{Findings and interpretations}

Based on the analysis of the collected data, the experiences of the participants with the emoji-based
UEQ were categorized as follows: (1) advantages of adopting the emoji-based UEQ, and (2) challenges of adopting the emoji-based UEQ. The interviewee's responses have been transcribed verbatim to relay to their responses accurately ${ }^{2}$.

\subsection{Advantages of adopting the emoji-based UEQ}

The survey participants shared several advantages of the adoption of emojis in UEQ. They deemed the use of emojis as positive because they made the survey easy to understand, fun, and engaging, as well as facilitated the decision-making process, language proficiency, and thinking style.

4.1.1 Easy to understand. The design of any survey plays a large role in determining the participant experience, as the design can have significant impact on how easily the survey may be understood. Applying the single-scale emoji format instead of the two columns of words used in the first version of the questionnaire improved participants' experiences, as described in the quotes below.

Second one is faster and easier to understand compared to the first one because in the first one I had to think between two different things while the second one had one aspect only. . .

The first survey took me longer because I had to think more about the two terms and where I would balance between those two, but this one I was only reacting to one term at a time.

I like the emojis better . . I knew exactly what you want me to do. There was no hesitation at all. It led you to the answer . . . instead of having two words at the side, it was just one word there and the faces[emoji]tell you what you think about it.

4.1.2 Fun and engaging. Another advantage is that some participants found it fun to interact with the emojis. For example, one participant, who indicated that she "always" used emojis, prefer the emoji-based UEQ because they were "more fun to do" and "it was clear." Another participant, whose emoji use was recorded as "most of the time," described her thoughts on introducing emojis into surveys as "I think using emojis is a good thing. It will not be complex at all." Another said, "it is more colorful and engaging."

\footnotetext{
2 "verbatim" quotes from survey participants may include poor grammar since some of the participants do not have English as their first language
} 
4.1.3 Facilitate the decision-making process. The simplicity of the process also aids some of the participants in decision-making. The single-step decision making meant that the survey required less time to complete, as described in the quotes below.

I feel the first version was more formal while filling out the survey whereas the second one was more fun and I think it expressed my feeling in more accurate way. . . which helped me to select faster compared to the first one.

It requires less brain work which I love . . . it is less stressful. . .

Further, one participant indicted that the facial emojis assisted the actual decision making.

It [emoji version] makes you have a direct impression and level of sadness or happiness. It is much easier for me.

4.1.4 Language proficiency. Participants were asked whether English was their native tongue. Those who answered "no" were asked to rate the proficiency of their English as follows: very well, well, not well, or not at all. Participants who identified as speaking English not well tended to prefer the emoji-based survey more than those who identified as speaking English well or very well. One participant who chose the "not well" label claimed that the emojis assisted her in completing the questionnaire.

. . . I think using emojis is probably more efficient that would have more accurate responses, especially that English is not my first language, so I found emoji useful for me.

4.1.5 Thinking style. During the interview, participants described their thinking processes as visual, systematic, and or mathematic. The data analysis revealed that those who identified as visual people preferred the emoji-based survey. One participant described the emoji version of the questionnaire as "...cool, and I am a visual person .. . I feel more comfortable compared to the numbers." Another participant who identified as a visual person said that "absorbing emoji in questionnaires is interesting. I would like to do more surveys if they have emoji."

\subsection{Challenges of the emoji-based UEQ}

The participants shared some of the challenges they faced in responding to the emoji-based survey. These participants stated that the emojis affected the quality of their experience in a negative way. They described the emojis as an informal and unprofessional way of communicating, as having too many possible interpretations, as distracting, as presenting toooptimistic results, and as more demanding in terms of effort and energy.

4.2.1 Emoji is used in informal and unprofessional communication. Participants were asked how often they used emojis in texting and/or on social media. For texting, some participants reported that their emoji use varied based on how close they were to the text recipient. One respondent described emoji use as "sometimes, depending on how close I am to the person," and another stipulated that it "depends on whom I am texting..." Other participants indicated that they preferred not to use emojis on social media because they felt that doing so affected their perceived image. One responded said she enjoyed using emojis when texting friends, "but I avoid using it in social media, because I advertised myself in social media as a professional person." One participant said that using emojis in a survey was unprofessional and informal, "the emoji survey feels sort of informal . . . it is a bit informal, and not professional."

4.2.2 Multiple interpretations of emoji characters. Some participants found criticism in that emojis can be interpreted to mean different things, which affects the accuracy of their use as well as the experience of filling out the survey that employs them.

I have a problem with the neutral face. . I I feel the neutral face is upset, partly negative . . . I avoided choosing the neutral because I think the neutral emoji is upset.

I think there is a problem with the last emoji, the one that is laughing. I think it would be better if you pick another one. . . I would choose another one to represent the happiness. .

The perceived meaning of a standalone emoji character varies among individuals, even when those individuals use the same platform [41, 42]. Clark's [43] psycholinguistics theory proposes that to decrease the probability of miscommunication incidents, the interpretation of an emoji must be standardized among those regarding it. This also requires that the participants know that they interpret emojis in the same way. 
4.2.3 Emojis are distracting. Some of the participants reported their concerns with the observation that the emojis were distracting.

It is overwhelming because I had to make sure about the emojis ... which one I am feeling.

It is distracting because it includes pictures that I needed to interpret.

...the emojis maybe because I don't use them. I don't even have a smart phone, but they were for me at first a little bit distracting. Instead of a clear-cut line 1 through 5, now you have to interpret what each of the emoji is saying...

4.2.4 Optimistic results. Some of the participants claimed that the presence of emojis in the questionnaire created a positive association, making the respondents more inclined to choose from the positive side of the emoji scale.

I know that I answered them differently. I would say when I filled out the one with emoji, I tended to give it a higher score. I feel like I did that...

I noticed that I chose more happy faces. . . I personally like or am attractive by happy faces. So, my answers did not really reflect my real or true feelings.

4.2.5 Demanding more effort and energy. Some of the participants reported their concerns that emojis added an additional level of effort in the cognitive decision-making process when selecting the final response to each question.

It was more emotionally investing in the second one, in which I spent slightly more time. . .

With emojis, I needed to think about the meaning then make the choice, but the first one was direct... I feel it is not that easy because I did the earlier faster than this... some people might be more familiar with emojis so they don't really need to think. But for me, I need to think if this means happy or not. Even though they are presented in the order of unsatisfied to happy. I still had to go through that thinking process.

Other participants compared responding to the emojis to hearing or reading words in a second language.

I feel they are little not that efficient to me because I had to go through extra steps of thinking. It is kind of like a second language... it is not direct.
"... It is like translation to another language".

Those who identified themselves as systematic or mathematic thinkers tended to dislike the emoji-based survey more than the visual thinkers. Some of these participants described their experience as follows:

I feel numbers better represent where I meant to put my answer.

I guess it depends on how people think ... some think more visually and some people think more like systematically or mathematically . . and so me being kind of more systematic, mathematic person, sometimes I like to see 1, 2, 3, 4, 5. But I think for a lot of other people the visual thing is definitely an advantage for a survey.

\section{Discussion}

Much of the current literature on emojis focuses on the interpretation and comparison of popular emoji characters across different platforms [41], or psychological assessments of human users [44, 45]. Such research is useful in developing an understanding of and applying emojis. If we apply the characteristics of emojis described by [19], emojis may be used in subjective assessments and opinion expression as a language-free alternative to items that usually require a certain level of fluency in a specific language.

Overall, this study aimed to investigate how the use of emojis in UEQs influence the experience of participants. Participants expressed advantages and/or challenges with the emoji version of the devised UEQ. Notably, differences in participant feedback were not only attributed to their experiences regarding the adoption of emoji, but also to the design of the questionnaire. The change from the semantic differential questionnaire (version 1) to the singledimension version seemed to affect participant experiences. One respondent compared the second version of the questionnaire to the first as follows:

I think it was a little bit challenging not having a word to compare it to, but in the same sense I think it was also an advantage because it sometimes can be confusing when you have opposite words and where does it fall in the middle.

Individual decision-making patterns also impacted the participant experience. Self-identified visual respondents' decision-making processes depended on feeling and intuition, so these participants tended to respond more positively to the emoji questionnaire. One participant explained this as follows: 
For the numbers, I was struggling to make decision. For example, [I think the mobile app] is understandable, [but] it is hard for me to select between 1 and 2... When I see [the emoji], I can directly have a sense that I am this happy [the mid-happy face] or that happy [the happiest face] when assessing certain item. I can link my level of agreement with the picture [emoji]

Participants who identified themselves as systematic or mathematical thinkers, in contrast, responded better to decision-making processes that followed a specific pattern (e.g., a defined scale). These respondents tended to be more concerned with the emoji questionnaire, partly because this version required the additional step of translating and transforming the emojis onto a five-point scale.

The first version [the semantic differential one] links to my brain, but the second one links to my heart.

The two versions used different parts of brain to make decisions.

As demonstrated, the decision-making patterns of the participants influenced their opinions on the adoption of emojis in UEQs. The results indicate that emoji questionnaires are not effective for people who make decisions based on systematically rational patterns. The adoption of emojis in UEQs, therefore, must depend on the characteristics of the target audience. The data analysis showed that age is not a significant factor in the way participants perceived the emoji-based UEQ, although several participants suggested that the emoji-based questionnaires could be helpful and more applicable to younger audiences who have yet to develop firm decision-making patterns.

It would be more appropriate [to conduct the emoji-based questionnaire] in millennials, since they have little experience in filling out surveys...

Some participants experienced confusion while filling out the emoji UEQ. For instance, one mentioned:

...if the question is negative, for example dull, if I choose the happy face does it means I agree with dull or I don't agree with it? So, it was a little bit confusing

At the end of the interviews, participants were asked whether they had any opinions on or disagreed with the emojis selected for the scale in the questionnaire. Many participants stated that the chosen neutral face $\odot$ did not convey neutrality well. These participants suggested using different emojis $(\odot$, $\odot$, (*) ) to replace the neutral face.

I think the neutral face doesn't mean the neutral for me...

One participant advised that the emojis selected for the scale should be as distinctive as possible to facilitate the direct and easy interpretation of their correct meaning.

\section{The level should be more distinctive and include more expression}

These responses will help future researchers design a more appropriate emoji-based UEQ for assessing UXs of mobile applications. The use of emojis to clarify the specific responses of questionnaire participants might be extended to a wide range of industries and applications. For example, one participant in the present study observed that emojis could help him better identify patient pain levels in his work as a physical therapist.

It depends on whom I am working with,
sometimes I used 1 to 10 scale. but I feel if a
person says 7 , it is subjective where everyone is
kind of different... while emojis narrow it down
to tell whether you are happy or you are okay
happy...

\section{Conclusion and implications}

Emojis, a growing phenomenon, have gained enormous popularity in interpersonal communication and emotional expression. However, a notable absent topic is the adoption of emojis in UEQs. The present study described how emojis can be used as a potential survey instrument to enhance the user experience when filling out UEQs. This study drew on semi-structured interview data to evaluate how emojis added or detracted from the participant experience. This exploratory case study contributes to the existing body of knowledge on emojis by presenting the detailed experiences of those who interacted with emoji-based UEQs. Using mobile-based emojis can also aid communication in various healthcare areas such as in mental healthcare therapy among young patients by facilitating the way they express their moods and allowing specialists to assess their mental distress [3]. Another implication is that emoji can be used to increase public health awareness. For instance, GE Healthcare used emojis in an educational YouTube video to explain breast density [46].

One limitation is that this work did not intend to validate the emoji questionnaire as a quantitative instrument. It will be an important distinction of future 
work to develop and validate its own emoji-based questionnaire with a truly random sample to assess user experiences, especially for mobile applications.

\section{References}

[1] J. B. Walther and K. P. D'Addario, "The impacts of emoticons on message interpretation in computer-mediated communication," Social Sci. Comput. Review, vol. 19, no. 3, pp. 324-347, 2001.

[2] X. Lu et al., "Learning from the ubiquitous language: an empirical analysis of emoji usage of smartphone users," In Proceedings of the 2016 ACM International Joint Conference on Pervasive and Ubiquitous Computing, Heidelberg, Germany, Sept. 2016, pp. 770-780.

[3] D. Donovan, "Mood, emotions and emojis: conversations about health with young people," Mental Health Practice, vol.20, no.2, pp.23, 2016.

[4] Kaye, L. K., Malone, S. A., \& Wall, H. J. Emojis: Insights, affordances, and possibilities for psychological science. Trends in cognitive sciences, 21(2), pp.66-68, 2017.

[5] International Organization for Standardization, ISO 9241210:2010. [Online]. Available: https:/www.iso.org/obp/ui/\#iso:std:iso:9241:-210:ed-1:v1:en

[6] B. Laugwitz et al., "Construction and evaluation of a user experience questionnaire," in Symposium of the Austrian HCI and Usability Engineering Group, Berlin, Nov. 2008, pp. 6376.

[7] P. K. Novak et al., "Sentiment of emojis," 2015. [Online]. Available:

http://journals.plos.org/plosone/article?id=10.1371/journal.po ne. 0144296

[8] R. Kelly and L. Watts. (2015). "Characterising the inventive appropriation of emoji as relationally meaningful in mediated close personal relationships". Experiences of Technology Appropriation: Unanticipated Users, Usage, Circumstances, and Design. [Online]. Available: http://opus.bath.ac.uk/46780/

[9] C. Taggart, New Words for Old: Recycling Our Language for the Modern World. London: Michael O'Mara Books Limited, 2015.

[10] H. Pohl et al., "Beyond just text: semantic emoji similarity modeling to support expressive

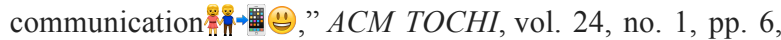
2017.

[11] Oxford University Press. (2016). Oxford Dictionaries word of the year is... [Online]. Available: http://blog.oxforddictionaries.com/2015/11/word-of-the-year2015-emoji.

[12] G. O'Brien. (2016). The Word on the Street is not a Word: It's An \& [Online]. Available: http://www.digitalamerica.org/the-word-on-the-street-is-nota-word-its-an-grace-obrien/

[13] J. N. Bavelas and N. Chovil, "Visible acts of meaning: an integrated message model of language in face-to-face dialogue," J. of Language and Social Psychology, vol. 19, no. 2, pp. 163-194, 2000

[14] M. Danesi, The Semiotics of Emoji: The Rise of Visual Language in the Age of the Internet. London: Bloomsbury Publishing, 2016.

[15] P. Ekman and W. V. Friesen, "The repertoire of nonverbal behavior: categories, origins, usage, and coding," Semiotica, vol. 1, pp. 49-98, 1969.

[16] M.L. Knapp, Nonverbal Communication in Human Interaction. New York: Rinehart and Winston, 1972, pp. 945

[17] M. Danesi, "Kinesics," Encyclopedia of Language and Linguistics, 2006, pp. 207-213.

[18] S. W. Littlejohn and K. A. Foss, Theories of Human Communication, 10th Edn. Long Grove, IL: Waveland Press, 2012.

[19] C. Durante, "Adapting nonverbal coding theory to mobile mediated communication: an analysis of emoji and other digital nonverbal," M.S. thesis, Dept. Commun. Stud., Liberty Univ.,

Virginia, Lynchburg, 2016.

[20] J. K. Burgoon et al., Nonverbal Communication: The Unspoken Dialogue 2nd Edn. New York, NY: McGraw-Hill, 1996.

[21] M. L. Knapp and J. A. Hall, "Elements of Nonverbal Communication, "in Nonverbal Communication in Human Interaction, 5th Edn. New York: Holt Rinehart and Winston, 2002, pp.61-71.

[22] B. Laurel. Computers As Theatre. Reading, MA: Addison-Wesley, 1993, pp. 14-22

[23] J. Dewey, Art as Experience. New York, NY: Perigee, 1980 , pp. 355.

[24] J. Forlizzi and S. Ford, "The building blocks of experience: an early framework for interaction designers," in Proceedings of the Conference on Designing interactive systems, Communications of the ACM, New York, 2000, pp. 419-423. 
[25] M. Hiltunen et al., Mobile User Experience. Finland: Edita Publishing Inc., 2002, pp. 214.

[26] M. Hassenzahl, "User experience (UX): towards an experiential perspective on product quality," in Proceedings of the 20th International Conference of the Association Francophone D'interaction Homme-Machine, vol. 339, New York, NY, 2008, pp. 11-15.

[27] E. Law et al., "Understanding, scoping and defining user experience: a survey approach," in Proceedings of the ACM SIGCHI Conference on Human Factors in Computing Systems, Boston, MA, 2009, pp. 719-728.

[28] A.P.O.S. Vermeeren et al., "User experience evaluation methods: current state and development needs," in Proceedings of Nordic Conference on Human-Computer Interaction, Reykjavik, Iceland, 2010, pp. 16-20.

[29] J. S. Dumas and M. C. Salzman. "Usability Assessment Methods." Reviews of Human Factors and Ergonomics, 2, pp. 109-144, (2006)

[30] W. Karwowski, International Encyclopedia of Ergonomics and Human Factors, 2nd Edn. Boca Raton, FL: Taylor \& Francis, 2006.

[31] T. Tullis and B. Albert, Measuring the User Experience: Collecting, Analyzing, and Presenting Usability Metrics. San Francisco, CA: Morgan Kaufmann, 2008.

[32] M. Schrepp et al., "Applying the user experience questionnaire (UEQ) in different evaluation scenarios," in International Conference of Design, User Experience, and Usability, Heraklion, Greece, 2014, pp. 383-392.

[33] C. E. Osgood et al., The Measurement of Meaning. Urbana, IL: University of Illinois, 1957.

[34] A. Bandura, "Social cognitive theory of mass communication," Media Psychology, vol. 3, pp. 265-299, 2001.

[35] A. Bandura and R. H. Walters, Social Learning and Personality Development, Vol. 14. New York, NY: Holt, Rinehart and Winston, 1963.

[36] A. Bandura, Social Learning Theory. Englewood Cliffs, NJ: Prentice Hall, 1977.

[37] J. E. Grusec, "Social learning theory and developmental psychology: the legacies of Robert Sears and Albert Bandura," Developmental Psychology, vol. 28, no. 5, pp. 776, 1992.

[38] A. Luszczynska and R. Schwarzer, "Social cognitive theory," in Predicting Health Behaviour, $2^{\text {nd }}$ ed. Buckingham, England: Open University Press, 2005, pp. 127-169.
[39] Emojiscore. (2017). Overview. [Online]. Available: http://www.emojiscore.com/overview/

[40] M. B. Miles and A. M. Huberman, Qualitative Data Analysis: An Expanded Sourcebook. Thousand Oaks: Sage Publications, 1994.

[41] H. J. Miller et al., "Blissfully happy or ready to fight: varying interpretations of emoji," in Tenth International AAAI Conference on Web and Social Media, Cologne, Germany, 2016, pp. 259-268.

[42] G. W. Tigwell and D. R. Flatla, "Oh that's what you meant! Reducing emoji misunderstanding," In Proceedings of the 18th International Conference on Human-Computer Interaction with Mobile Devices and Services Adjunct, Florence, Italy, 2016, pp. 859-866.

[43] H. H. Clark, Using Language. Cambridge: Cambridge University Press, 1996.

[44] A. C. K. Lee et al., "The smiley as a simple screening tool for depression after stroke: a preliminary study," Int. J. of Nursing Stud., vol. 45, no. 7, pp. 1081-1089, 2008.

[45] D. Marengo et al., "Assessing personality using emoji: an exploratory study," Personality and Individual Differences, vol. 112, pp. 74-78, 2017.

[46] D. J. Skiba, "Face with tears of joy is word of the year: are emoji a sign of things to come in health care?," Nursing education perspectives, vol.37, no.1, pp.56-57, 2016.

\section{Appendix A: Semi-structured interview questions}

Part 1

1. What was your overall feeling when you were filling out the questionnaire?

2. What problems or challenges did you experience when you filled out the UEQ, if any?

3. Did you find it clear and easy to understand? Explain why?

Part 2

1. What was your overall feeling when you were filling out the questionnaire?

2. What differences did you experience when filling out the $2^{\text {nd }}$ version compared with the first one?

3. Is the emoji-based questionnaire unnecessarily complex? Why?

4. Do you have any comments on using emoji to answer a questionnaire? 


\section{Appendix B: UEQ (Version 1)}

Please assess the Mobile application now by ticking one circle per line.

\begin{tabular}{|c|c|c|c|c|c|c|c|}
\hline & 1 & 2 & 3 & 4 & 5 & & \\
\hline annoying & 0 & 0 & 0 & 0 & 0 & enjoyable & 1 \\
\hline not understandable & O & O & O & O & 0 & understandable & 2 \\
\hline creative & O & O & O & O & O & dull & 3 \\
\hline valuable & O & O & O & O & 0 & unvaluable & 4 \\
\hline boring & O & O & O & $\mathrm{O}$ & $\mathrm{O}$ & exciting & 5 \\
\hline not interesting & O & 0 & O & 0 & 0 & interesting & 6 \\
\hline unpredictable & O & O & O & O & O & predictable & 7 \\
\hline fast & O & 0 & O & O & 0 & slow & 8 \\
\hline obstructive & O & O & O & O & O & supportive & 9 \\
\hline good & O & 0 & O & O & O & bad & 10 \\
\hline complicated & O & O & O & O & O & easy & 11 \\
\hline unlikable & O & O & 0 & O & 0 & pleasing & 12 \\
\hline usual & O & O & O & O & O & leading edge & 13 \\
\hline secure & 0 & 0 & 0 & 0 & 0 & not secure & 14 \\
\hline motivating & O & 0 & O & O & O & demotivating & 15 \\
\hline meets expectations & O & 0 & O & O & 0 & does not meet expectations & 16 \\
\hline inefficient & $\mathrm{O}$ & 0 & O & O & 0 & efficient & 17 \\
\hline impractical & O & O & O & O & O & practical & 18 \\
\hline organized & O & O & O & O & O & cluttered & 19 \\
\hline attractive & O & O & O & O & O & unattractive & 20 \\
\hline friendly & O & O & O & O & O & unfriendly & 21 \\
\hline conservative & 0 & 0 & O & 0 & 0 & innovative & 22 \\
\hline
\end{tabular}

\section{Appendix C: Emoji-based UEQ (Version 2)}

Please assess the Mobile application now by selecting an emoji per line.

\begin{tabular}{|c|c|c|c|c|c|c|}
\hline enjoyable & 8 & $\Leftrightarrow$ & 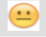 & (c) & $\theta$ & 1 \\
\hline understandable & 8 & $\therefore$ & 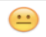 & (4) & $\Leftrightarrow$ & 2 \\
\hline creative & 8 & 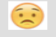 & 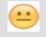 & (4) & $\theta$ & 3 \\
\hline valuable & 8 & 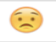 & $\rightleftharpoons$ & (ن) & $\therefore$ & 4 \\
\hline exciting & 8 & $\because$ & $\rightleftharpoons$ & (c) & $\Leftrightarrow$ & 5 \\
\hline interesting & 8 & $\Leftrightarrow$ & $\rightleftharpoons$ & (c) & $\Leftrightarrow$ & 6 \\
\hline predictable & 8 & $\Leftrightarrow$ & 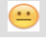 & (c) & $\Leftrightarrow$ & 7 \\
\hline fast & 8 & $\therefore$ & $\because$ & (0) & $\Leftrightarrow$ & 8 \\
\hline supportive & 8 & $\therefore$ & $\because$ & (c) & $\theta$ & 9 \\
\hline good & 8 & $\therefore$ & 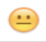 & (c) & $\Leftrightarrow$ & 10 \\
\hline easy & 8 & $\because$ & 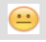 & (3) & $\theta$ & 11 \\
\hline leading edge & 8 & 9 & 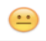 & (4) & 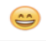 & 12 \\
\hline pleasant & 8 & $\because$ & (-) & (c) & $\Leftrightarrow$ & 13 \\
\hline secure & 8 & $\Leftrightarrow$ & $\rightleftharpoons$ & (c) & $\Leftrightarrow$ & 14 \\
\hline motivating & 8 & 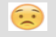 & $\rightleftharpoons$ & (c) & $\Leftrightarrow$ & 15 \\
\hline meets expectations & 8 & $\Leftrightarrow$ & 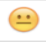 & (c) & $\Leftrightarrow$ & 16 \\
\hline efficient & 8 & $\%$ & 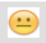 & (9) & $\Leftrightarrow$ & 17 \\
\hline practical & 8 & $\Leftrightarrow$ & 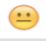 & (3) & $\Leftrightarrow$ & 18 \\
\hline organized & 8 & $\therefore$ & (-) & (4) & $\Leftrightarrow$ & 19 \\
\hline attractive & 8 & $\Leftrightarrow$ & 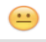 & (4) & $\Leftrightarrow$ & 20 \\
\hline friendly & (2) & $\Leftrightarrow$ & 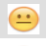 & (4) & $\Leftrightarrow$ & 21 \\
\hline innovative & 8 & $\Leftrightarrow$ & 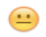 & (4) & $\Leftrightarrow$ & 22 \\
\hline
\end{tabular}

